\title{
On the exploitation of the Eshelby stress in isothermal and adiabatic conditions
}

\author{
Gérard A. Maugin ${ }^{\mathrm{a}}$ and Arkadi Berezovski ${ }^{\mathrm{b}}$ \\ a Laboratoire de Modélisation en Mécanique, UMR CNRS 7607, Université Pierre et Marie \\ Curie, case 162, place Jussieu 4, 75252 Paris cedex 05, France; gam@ ccr.jussieu.fr \\ b Centre for Nonlinear Studies, Institute of Cybernetics at Tallinn University of Technology, \\ Akadeemia tee 21, 12618 Tallinn, Estonia; Arkadi.Berezovski@cs.ioc.ee
}

Received 15 January 2007

\begin{abstract}
This note emphasizes the particular role played by the quantity "entropy multiplied by temperature" in the formulation of canonical thermomechanics either in the bulk or at singular surfaces, especially at shock waves and phase transition fronts, but more generally when working hypotheses of adiabatic or isothermal behaviour must be selected.
\end{abstract}

Key words: Eshelby stress, free energy, internal energy, shock waves, phase-transition fronts.

\section{INTRODUCTION}

The material stress tensor, known as the Eshelby stress, plays a fundamental role in the study of forces, which are driving defects and field singularities in thermomechanics. Since its definition $\left[{ }^{1}\right]$ involves both the potential energy and the stress, and the corresponding driving force has a thermodynamic nature, the thermodynamic conditions in which we apply this concept are most relevant, in particular, whether isothermal or adiabatic conditions prevail during the motion of the said defects or singularities. This question has been discussed in $\left.{ }^{2-5}\right]$ and is revisited in greater detail in this contribution. Indeed, if $\theta$ and $S$ are temperature and entropy per unit reference volume, and $W$ and $E$ are free and internal energies, also per unit reference volume, then we usually have the following relations:

$$
W(., \theta)=E(., S)-S \theta, \quad S=-\frac{\partial W}{\partial \theta}, \quad \theta=\frac{\partial E}{\partial S}>0,
$$


where the first one is a celebrated Legendre transformation. Replacing the dots by the deformation gradient $\mathbf{F}$ of the finite strain theory in expression (1), we have the standard theory of thermoelasticity, which is complemented by Fourier's law of heat conduction. In the sequel the transformation term $(S \theta)$ will be shown to play a more important part than usually assumed. This is due to its appearance as a natural basic energy (Section 2), and also due to its role in the definition of various Eshelby-like tensors in the bulk (Section 3) or at a discontinuity surface (Section 4).

\section{CANONICAL BALANCE LAWS OF MOMENTUM AND ENERGY}

In all field theories of which continuum mechanics offers the paragon, there are balance laws (e.g., mass, linear momentum, energy) and canonical conservation equations. In a most general setting, it was recently shown in continuum mechanics that a set of consistent space-like and time-like conservation laws of momentum and energy is given by the following two equations (in the absence of body force and material inhomogeneities) $\left[{ }^{6}\right]$ :

$$
\frac{\mathrm{d} \mathbf{P}}{\mathrm{d} t}-\nabla_{R} \cdot \mathbf{b}=\mathbf{f}^{\text {int }}, \quad \frac{\mathrm{d}(S \theta)}{\mathrm{d} t}+\nabla_{R} \cdot \mathbf{Q}=h^{\mathrm{int}},
$$

where

$$
\begin{gathered}
\mathbf{P}=-\rho_{0} \mathbf{v} \cdot \mathbf{F}, \quad \mathbf{b}=-\left(L_{W} \mathbf{1}_{R}+\mathbf{T F}\right), \\
\mathbf{f}^{\text {int }}=\mathbf{T}:\left(\nabla_{R} \mathbf{F}\right)^{T}-\left.\nabla_{R} W\right|_{\text {impl }}, \quad h^{\text {int }}=\mathbf{T}: \dot{\mathbf{F}}-\left.\frac{\partial W}{\partial t}\right|_{X} .
\end{gathered}
$$

Here, $\mathbf{1}_{R}$ is the unit tensor in material space, a superimposed dot denotes the material time derivative, the subscript "impl" means the gradient taken only through the field variables, and

$$
\mathbf{v}=\left.\frac{\partial \overline{\mathbf{x}}}{\partial t}\right|_{X}, \quad \mathbf{F}=\left.\frac{\partial \overline{\mathbf{x}}}{\partial \mathbf{X}}\right|_{t}
$$

are the physical velocity and deformation gradient if $\mathbf{x}=\overline{\mathbf{x}}(\mathbf{X}, t)$ is the direct motion; $\rho_{0}$ is the matter density at the reference configuration, $\mathbf{P}$ is the canonical (or material) momentum, $\mathbf{b}$ is the Eshelby material stress tensor, $L_{W}=K-W$, $K=\rho_{0} \mathbf{v}^{2} / 2, \mathbf{Q}$ is the material heat flux, and $\mathbf{f}^{\text {int }}$ and $h^{\text {int }}$ are "internal sources" of momentum and energy of unknown expression before a postulate of an energy expression (for other notations see $\left[{ }^{6}\right]$ ). For instance, in standard thermoelasticity, we select $W=\bar{W}(\mathbf{F}, \theta)$ as being the free energy. Then Eqs (2) reduce to

$$
\frac{\mathrm{d} \mathbf{P}}{\mathrm{d} t}-\nabla_{R} \cdot \mathbf{b}_{W}=\mathbf{f}^{\mathrm{int}}:=S \nabla_{R} \theta, \quad \frac{\mathrm{d}(S \theta)}{\mathrm{d} t}+\nabla_{R} \cdot \mathbf{Q}=h^{\mathrm{int}}:=S \dot{\theta},
$$

where

$$
\mathbf{b}_{W}=-(K-\bar{W}(\mathbf{F}, \theta)) \mathbf{1}_{R}-\mathbf{T}(\mathbf{F}, \theta) \mathbf{F}, \quad \mathbf{T}=\left.\frac{\partial \bar{W}(\mathbf{F}, \theta)}{\partial \mathbf{F}}\right|_{\theta},
$$


while Eq. $(6)_{2}$ is none other than the classical heat propagation equation

$$
\theta \dot{S}+\nabla_{R} \cdot \mathbf{Q}=0, \quad S=-\left.\frac{\partial \bar{W}}{\partial \theta}\right|_{\mathbf{F}} .
$$

\section{BULK CASE}

Had we chosen to work with the internal energy $E$ per unit reference volume such that (cf. Eq. (1))

$$
E=\bar{E}(\mathbf{F}, S), \quad \theta=\left.\frac{\partial \bar{E}}{\partial S}\right|_{\mathbf{F}}, \quad \mathbf{T}=\left.\frac{\partial \bar{E}}{\partial \mathbf{F}}\right|_{S}
$$

for a materially homogeneous thermoelastic material, we would have obtained the (non)conservation of material momentum in the following form:

$$
\begin{gathered}
\frac{\mathrm{d} \mathbf{P}}{\mathrm{d} t}-\nabla_{R} \cdot \mathbf{b}_{E}=\mathbf{f}_{S}^{\mathrm{th}}, \\
\mathbf{b}_{E}=-\left(L_{E} \mathbf{1}_{R}+\mathbf{T F}\right), \quad L_{E}=K-\bar{E}(\mathbf{F}, S), \quad \mathbf{f}_{S}^{\mathrm{th}}=-\theta \nabla_{R} S .
\end{gathered}
$$

In particular, we note the following relationship

$$
\mathbf{f}^{\text {th }}:=\mathbf{f}_{\theta}^{\text {th }}=\mathbf{f}_{S}^{\text {th }}+\nabla_{R} \cdot\left(S \theta \mathbf{1}_{R}\right) .
$$

This has consequences at discontinuity surfaces, which may be homothermal or essentially considered in the adiabatic framework although related to a dissipative progress.

\section{THE CASE OF SINGULAR SURFACES: SHOCK WAVES AND PHASE-TRANSITION FRONTS}

In the case of the propagating singular surface $\mathcal{S}$ (with unit oriented normal $\mathbf{N}$ ), entirely described in the material framework, it is clear that the presence of such a surface breaks the translational invariance on the material manifold, since the material will in general have acquired different material properties on both sides of the surface. Accordingly, the central equation - the one which will deliver the driving force on the singular surface - is the jump relation associated with the regular bulk equation of material (or canonical) linear momentum, because this equation contains the "material force" generated by a material displacement of $\mathcal{S}$ on the material manifold. The classical jump relations of mass, linear (physical) momentum, and energy at $\delta$ read (cf. $\left.\left[{ }^{7}\right]\right)$

$$
(\overline{\mathbf{V}} \cdot \mathbf{N})\left[\rho_{0}\right]=0,
$$




$$
\begin{gathered}
\mathbf{N} \cdot[\mathbf{T}+\overline{\mathbf{V}} \otimes \mathbf{p}]=\mathbf{0}, \\
\mathbf{N} \cdot[\overline{\mathbf{V}} H+\mathbf{T} \mathbf{v}-\mathbf{Q}]=0,
\end{gathered}
$$

where $\overline{\mathbf{V}}$ is the material velocity of $\mathcal{S}, H=K+E$, i.e., the sum of kinetic and internal energies, $\mathbf{p}=\rho_{0} \mathbf{v}$ is the linear momentum. These equations do not exhibit source terms. This is not the case with the jump relations associated with material momentum and entropy (canonical (non)conservation laws):

$$
\begin{aligned}
& \mathbf{N} \cdot[\mathbf{b}+\overline{\mathbf{V} \otimes \mathbf{P}}]+\mathbf{f}_{\mathcal{S}}=\mathbf{0}, \\
& \mathbf{N} \cdot\left[\overline{\mathbf{V}} S-\frac{\mathbf{Q}}{\theta}\right]-\sigma_{\mathcal{S}}=0,
\end{aligned}
$$

with the constraint (second law of thermodynamics at $\mathcal{S}$ )

$$
\sigma_{\mathcal{S}} \geq 0
$$

Here source terms $\mathbf{f}_{\mathcal{S}}$ and $\sigma_{\mathcal{S}}$ are unknown, as well as $\overline{\mathbf{V}}$ ! Accordingly, the interesting relationship is the one that relates the unknown driving force $\mathbf{f}_{\mathcal{S}}$ and the equally unknown (but non-negative) surface entropy source $\sigma_{\mathcal{S}}$. If the theory is consistent, these cannot be entirely independent. The consistency condition looked for in fact allows one to close the system of phenomenological equations at $\mathcal{S}$ in compliance with the second law.

\subsection{Shock waves}

For classical shock waves (in the so-called inconsistent theory, where a dissipative interface across which entropy grows is supposed to connect two regions nonetheless in the adiabatic regime; $\mathbf{Q}=\mathbf{0}$ ), one sets

$$
\mathbf{f}_{\mathcal{S}}=0, \quad \forall \overline{\mathbf{V}} \neq 0,
$$

and there remains the trivial relation

$$
\sigma_{\mathcal{S}}=[S](\overline{\mathbf{V}} \cdot \mathbf{N}) \geq 0,
$$

which tells in which direction (with respect to $\mathbf{N}$ ) the wave front moves to guarantee an increase in entropy, while one has to specify in terms of which energy $\mathbf{f}_{\mathcal{S}}$ is defined. Indeed, Eq. (16) can be expressed from the start in terms of $\mathbf{b}_{W}$ or $\mathbf{b}_{E}$. Accordingly, we should write either

$$
\mathbf{N} \cdot\left[\mathbf{b}_{W}+\overline{\mathbf{V}} \otimes \mathbf{P}\right]+\mathbf{f}_{\mathcal{S}, W}=\mathbf{0}
$$

or

$$
\mathbf{N} \cdot\left[\mathbf{b}_{E}+\overline{\mathbf{V}} \otimes \mathbf{P}\right]+\mathbf{f}_{S, E}=\mathbf{0}
$$


with

$$
\mathbf{f}_{\mathcal{S}, W}=\mathbf{f}_{\mathcal{S}, E}+\mathbf{N}[S \theta],
$$

while the entropy jump relation (17) can be rewritten as

$$
\bar{V}_{N}[S]-\mathbf{N} \cdot[\mathbf{Q}]\left\langle\frac{1}{\theta}\right\rangle-\mathbf{N} \cdot\langle\mathbf{Q}\rangle\left[\frac{1}{\theta}\right]=\sigma_{\mathcal{S}} .
$$

Here the symbols $[\ldots]$ and $\langle\ldots\rangle$ denote, respectively, the jump and mean value of the enclosed quantity at $\mathcal{S}$.

Both adiabatic and non-adiabatic conditions can be summarized in the "product equation" (not a physical equation; i.e., either one of the factors in the left-hand side may vanish $\left.\left[{ }^{2}\right]\right)$

$$
\mathbf{Q}[\theta]=\mathbf{0} .
$$

Therefore, necessarily

$$
\mathbf{N} \cdot[\mathbf{Q}]\left(\left\langle\frac{1}{\theta}\right\rangle-\frac{1}{\langle\theta\rangle}\right)=0 \quad \text { and } \quad \mathbf{N} \cdot\langle\mathbf{Q}\rangle\left[\frac{1}{\theta}\right]=0 .
$$

Substituting the normal jump of $\mathbf{Q}$ from Eq. (15), taking account of (16a) and (22) and the expression of the power expended by $\mathbf{f}_{\mathcal{S}, W}$ in the material motion $\overline{\mathbf{V}}$, we obtain that

$$
\sigma_{\mathcal{S}}=\frac{1}{\langle\theta\rangle}\left(\mathbf{f}_{\mathcal{S}, W}-\mathbf{N}\langle S\rangle[\theta]\right) \cdot \overline{\mathbf{V}},
$$

which provides the effective driving force acting on $\mathcal{S}$ (i.e., the factor of $\overline{\mathbf{V}}$ ). A dual formula involving the jump of entropy was obtained in $\left[{ }^{7}\right]$. On account of (21), we have thus

$$
\sigma_{\mathcal{S}}=\frac{1}{\langle\theta\rangle} \mathbf{f}_{\mathcal{S}, E} \cdot \overline{\mathbf{V}}+[S](\overline{\mathbf{V}} \cdot \mathbf{N}),
$$

and we have to compute

$$
\mathbf{f}_{\mathcal{S}, E} \cdot \overline{\mathbf{V}}=-[E(\overline{\mathbf{V}} \cdot \mathbf{N})-\langle\mathbf{N} \cdot \mathbf{T}\rangle \cdot \mathbf{F} \overline{\mathbf{V}}] .
$$

Projected onto the unit normal $\mathbf{N}$, the first of (19) then is none other than the celebrated Hugoniot equation of the shock-wave theory, i.e.,

$$
\operatorname{Hugo}_{\mathrm{sw}}=[E-\langle\mathbf{N} \cdot \mathbf{T}\rangle \cdot \mathbf{F N}]=0 .
$$

In these conditions we check that $\mathbf{f}_{\mathcal{S}, W}=\mathbf{N}[S \theta]$ in agreement with (21), while (19) holds good. 


\subsection{Phase-transition fronts}

For coherent phase-transition fronts for which

$$
[\mathbf{V}]=\mathbf{0}, \quad[\theta]=0,
$$

across $\mathcal{S}$, the above-given formula (25) reduces to

$$
\sigma_{\mathcal{S}}=\frac{\mathbf{f}_{\mathcal{S}} \cdot \overline{\mathbf{V}}}{\theta_{\mathcal{S}}}=\frac{f_{\mathcal{S}} \bar{V}_{N}}{\theta_{\mathcal{S}}} \geq 0
$$

where $\theta_{\mathcal{S}}$ is the value of $\theta$ at $\mathcal{S}, \bar{V}_{N}=\overline{\mathbf{V}} \cdot \mathbf{N}$ is the normal speed of $\mathcal{S}$, and the scalar surface driving force $f_{\mathcal{S}}$ is given by

$$
f_{\mathcal{S}}=-\mathrm{Hugo}_{\mathrm{pt}}, \quad \mathrm{Hugo}_{\mathrm{pt}}=[W-\langle\mathbf{N} \cdot \mathbf{T}\rangle \cdot \mathbf{F N}],
$$

and is generally not zero (it is zero for the nondissipative Landau theory of phase transitions where the vanishing of $f_{\mathcal{S}}$ is a mathematical statement akin - in the appropriate state space - to the "Maxwell's rule of equal areas" in the construction of the so-called Maxwell line).

\section{CONCLUSIONS}

Equations (28) and (31) illustrate perfectly the need for distinguishing between internal and free energies. They emphasize the use of one or the other, depending on the thermal conditions of the considered process across the wave front.

Another way to derive these relationships at $\mathcal{S}$ has been developed by one of the authors $\left[{ }^{7}\right]$ by introducing the notion of a single scalar quantity, a generating function or Massieu thermodynamic potential $M$ from which both $f_{\mathcal{S}}$ and $\sigma_{\mathcal{S}}$ are consistently derived. For this, we refer the readers to the original work. In conclusion, we note the recurring appearance of the product term $S \theta$ in both bulk equations and jump relations, in particular in the canonical expression $(2)_{2}$ of the energy balance, in the relationship (12), and similar jump relations (e.g., Eq. (21)), and the reduced expression of $\mathbf{f}_{\mathcal{S}, W}$ in the shock wave case (adiabatic conditions).

\section{ACKNOWLEDGEMENT}

Support of the Estonian Science Foundation under grant No. 5765 (A. B.) is gratefully acknowledged. 


\section{REFERENCES}

1. Maugin, G. A. Material Inhomogeneities in Elasticity. Chapman and Hall, London, 1993.

2. Abeyaratne, R. and Knowles, J. K. A note on the driving traction acting on a propagating interface: adiabatic and non-adiabatic processes of a continuum. ASME J. Appl. Mech., 2000, 67, 829-831.

3. Maugin, G. A. Remarks on the Eshelbian thermomechanics of materials. Mech. Res. Commun., 2002, 29, 537-542.

4. Abeyaratne, R., Bhattacharya, K. and Knowles, J. K. Strain-energy functions with local minima: modeling phase transformations using finite thermoelasticity. In Nonlinear Elasticity: Theory and Application (Fu, Y. B. and Ogden, R. W., eds). Cambridge University Press, U.K., 2001, 433-490.

5. Berezovski, A. and Maugin, G. A. On the thermodynamic conditions at moving phasetransition fronts in thermoelastic solids. J. Non-Equilib. Thermodyn., 2004, 29, 37-51.

6. Maugin, G. A. On canonical equations of continuum thermomechanics. Mech. Res. Commun., 2006, 33, 705-710.

7. Maugin, G. A. On shock waves and phase-transition fronts in continua. ARI, 1998, 50, $141-150$.

\section{Eshelby pinge kasutamine isotermilistel ja adiabaatilistel tingimustel}

\section{Gérard A. Maugin ja Arkadi Berezovski}

On rõhutatud erilist osa, mis on suurusel "entroopia korrutatud temperatuuriga" kanoonilises termomehaanikas kas paljudel või üksikutel pindadel, eriti lööklainel ja faasiülemineku frondil, aga veel üldisemalt juhul, kui tuleb valida adiabaatilise või isotermilise käitumise tööhüpoteesi vahel. 